\title{
SEGURIDAD DE LA HISTERECTOMÍA TOTAL POR LAPAROSCOPIA ENTRE EL 2007 Y 2017 EN UN HOSPITAL DE ALTA COMPLEJIDAD, PEREIRA, COLOMBIA. ESTUDIO DE COHORTE
}

\author{
Safety of total laparoscopic hysterectomy in a high \\ complexity Hospitall in Pereira, Colombia, between \\ 2007 and 2017. Cohort study \\ Claudia Bastidas-Guarín, MD, Msc ${ }^{1,2}$; Claudia Patricia Zambrano-Moncayo, MD $^{1,2}$; \\ Jorge Darío López-Isanoa, $\mathrm{MD}^{1,2}$; José Duván López-Jaramillo, $M D^{1,2}$; \\ Ana Lucía Herrera-Betancourt, ${ }^{1,2}$; Angélica Cuello-Salcedo, $\mathrm{MD}^{1,2}$; \\ Eliana Gutiérrez-Calderón, Msc ${ }^{2,3}$; Danny Leandro Piedrahíta-Gutiérrez, MD ${ }^{1,2}$; \\ Juan Diego Villegas-Echeverri, $M D^{1},{ }^{2}$ \\ Recibido: 14 de abril de 2020/Aceptado: 25 de agosto de 2020
}

\section{RESUMEN}

Objetivo: objetivo describir las complicaciones intra y posoperatorias de la histerectomía total por laparoscopia (HTL) para patología benigna del útero y hacer una comparación con otros hospitales que tienen altos volúmenes de HTL.

Materiales y métodos: estudio de cohorte retrospectivo en mujeres que se sometieron a HTL por patología ginecológica benigna entre 2007 y 2017 en una institución privada de alta complejidad, que atiende población perteneciente al régimen contributivo y subsidiado por el Estado en el Sistema General de Seguridad Social, en Pereira, Colombia, por el grupo de cirujanos del centro de entrenamiento ALGIA. Muestreo consecutivo.

* Correspondencia: Claudia Bastidas Guarín, Calle 19 43g-155, Torre 2, apto.107, Ciudad del Río, Medellín (Colombia). Claubasti01@hotmail.es Unidad de laparoscopia ginecológica avanzada y dolor pélvico - ALGIA, Pereira (Colombia).

Clínica Comfamiliar, Pereira (Colombia).

Fundación Universitaria Área Andina, Pereira (Colombia).
Se describen la características sociodemográficas y clínicas basales, los hallazgos intraoperatorios y las complicaciones intra y posoperatorias. Se usó estadística descriptiva.

Resultados: en el periodo de estudio se incluyeron 1.350 pacientes. El tiempo quirúrgico fue de $95 \mathrm{~min}(\mathrm{DE} \pm 31)$, el sangrado quirúrgico $88 \mathrm{cc}$ (DE \pm 66 ), el peso promedio del útero fue de 236 $\mathrm{g}$ (DE \pm 133). El 96,5\% de las pacientes fueron evaluadas entre los 30 y 45 días. El 3,48\% de las pacientes tuvieron complicaciones menores y el 2,5\% complicaciones mayores. Tres pacientes requirieron conversión a laparotomía (0,23\%). No hubo mortalidad en la muestra estudiada.

Conclusión: la HTL es un procedimiento seguro y la tasa de complicaciones es similar a la de los mejores estándares internacionales. Es importante que se sigan haciendo estudios prospectivos con criterios objetivos de evaluación para comparar el desempeño de los diferentes grupos e instituciones que ofrecen procedimientos quirúrgicos y entrenamiento. 
Palabras clave: histerectomía; laparoscopia; complicaciones.

\section{ABSTRACT}

Objective: To describe intra- and postoperative complications of total laparoscopic hysterectomy (TLH) for benign uterine pathology and to compare with other hospitals with large volumes of laparoscopic hysterectomies.

Materials and Methods: Retrospective cohort study of women who underwent TLH for benign gynecological pathology between 2007 and 2017 in a private high complexity institution that serves populations covered by contributive and state-subsidized insurance in Pereira, Colombia. The procedures were performed by the group of surgeons of the ALGIA training center. Consecutive sampling was used. Sociodemographic and baseline clinical characteristics, intra-operative findings and intraand post-operative complications are described. Descriptive statistics were used.

Results: A total of 1.350 patients were included during the study period. Surgical time was 95 minutes ( $\mathrm{SD} \pm 31$ ), blood loss $88 \mathrm{cc}(\mathrm{SD} \pm 66)$, and average uterine weight was $236 \mathrm{~g}(\mathrm{SD} \pm 133)$; $96.5 \%$ of the patients were assessed within the next 30 to 45 days; $3.48 \%$ had minor complications and 2.5\% had major complications; 3 patients were converted to laparotomy. There were no deaths in the study sample.

Conclusion: TLH is a safe procedure and the rate of complications is similar to the best international standards. Further prospective studies using objective criteria are important in order to compare the performance of different groups and institutions offering surgical procedures and training.

Key words: Hysterectomy; laparoscopy; complications.

\section{INTRODUCCIÓN}

La histerectomía es uno de los procedimientos quirúrgicos más realizados y su ruta ha tenido cambios
(1-3) desde la descripción de la vía laparoscópica hace más de 20 años (4). Esto se explica dado que la histerectomía total por laparoscopia (HTL) ha tenido mucha acogida entre los cirujanos por su efectividad, seguridad para las pacientes, menor tiempo de retorno a las actividades e incapacidad médica más corta, aunque puede tomar un mayor tiempo quirúrgico (5). Una revisión del Grupo Cochrane que comparó la HL con la histerectomía abdominal (HA) en 520 mujeres en 6 estudios, mostró que el tiempo de regreso a las actividades normales podría ser más corto en el grupo de HTL comparada con la hecha por laparotomía, con una disminución media (DM) de -13,6 días (IC 95 \%: -15,4 a -11,8), aunque también mostró más lesiones de vejiga en la HL que la HA en 2.400 mujeres (odds ratio $[\mathrm{OR}]=2,4$; IC $95 \%$ : 1,2-4,8), sin embargo, la evidencia fue calificada de baja certeza en la estimación de efecto. En esta revisión no se evidenciaron diferencias entre la HL y la HV (1,440 mujeres y 16 estudios) (6). Por otra parte, Dedden, en una revisión sistemática de estudios de cohorte prospectivos y retrospectivos, informa una frecuencia de reingreso del $4 \%$, una frecuencia de complicaciones menores que varió entre el 4,3 y 7,3\%, y complicaciones mayores entre el 0,7 y 3,6\% en estudios prospectivos y retrospectivos respectivamente (7). Por otra parte, se ha informado una frecuencia de complicaciones menores del 3,3\% y una frecuencia de transfusión intraoperatoria del $16 \%$ (8). Se ha descrito que en Finlandia la frecuencia de complicaciones disminuyó con la experiencia del equipo quirúrgico; así, en 1996 la frecuencia para la HL era del $17 \%$ y pasó a $15,5 \%$ en 2006, y la frecuencia de lesiones en órganos adyacentes bajó del 2,8 al 1,7 \% en este periodo (9). Otro factor asociado a las complicaciones es la participación de cirujanos en entrenamiento en las cirugías, lo cual se ha asociado a más complicaciones mayores en la histerectomía vaginal (3,3\% cuando participan cirujanos en entrenamiento frente a 2,3\% cuando no participan cirujanos en entrenamiento), mas no 
en la HL (3,0 frente a 2,9\% respectivamente), pero sí hay aumento de complicaciones menores tanto en HV (7,3\% frente a 5,4\%), como en la HL (5,9 frente a 4,3) respectivamente (10). Dado el incremento en la frecuencia de la HTL es importante medir la calidad de las instituciones para ofrecer este procedimiento y, con este fin, se ha diseñado un instrumento que toma en cuenta: el tiempo quirúrgico, la pérdida sanguínea durante el procedimiento y las complicaciones (11), lo que permite hacer comparaciones entre los diferentes grupos de ginecólogos laparoscopistas.

A pesar de las ventajas de la histerectomía por vías de mínimo acceso, publicadas en la literatura, la vía abierta todavía es la ruta de elección, sobre todo en los países de bajos y medianos ingresos; las razones parecen estar asociadas a los costos pero, además, se ha sugerido que se requiere incrementar la investigación en materiales menos costosos, en nuevos métodos de entrenamiento y en la seguridad de esta cirugía mínimamente invasiva en estos contextos (12).

En nuestro país hay poca evidencia publicada respecto al desempeño de grupos con experiencia de los centros de entrenamiento en este tipo de cirugía mínimamente invasiva. Es necesario publicar los resultados quirúrgicos de histerectomía laparoscópica de dichos centros del país y compararlos con otras instituciones a nivel regional y mundial en términos de seguridad. Este trabajo tiene como objetivo describir las complicaciones intra y posoperatorias de la histerectomía total por laparoscopia (HTL) en una cohorte de 10 años, en pacientes con patología ginecológica benigna, en un centro de entrenamiento de cirugía mínimamente invasiva y dolor pélvico crónico, y hacer una comparación con las descritas por otros hospitales que tienen altos volúmenes de histerectomía laparoscópica para patología benigna del útero.

\section{MATERIALES Y MÉTODOS}

Diseño y población. Cohorte retrospectiva. Se incluyeron mujeres con indicación de histerectomía por sangrado uterino anormal, miomatosis uterina, dolor pélvico crónico, adenomiosis, endometriosis, hiperplasia endometrial sin atipia, prolapso genital, que fueron intervenidas por vía laparoscópica entre enero de 2007 y diciembre de 2017, en la clínica Comfamiliar, por los especialistas integrantes de la Unidad de Laparoscopia Ginecológica Avanzada y Dolor Pélvico - ALGIA. Se excluyeron las pacientes llevadas a cirugía por una indicación oncológica, diagnosticadas antes de la cirugía o confirmadas en el resultado final de patología. Esta clínica atiende población perteneciente al régimen contributivo y subsidiado por el Estado en el Sistema de Seguridad social en Colombia. Se hizo muestreo consecutivo.

Procedimiento. Las pacientes fueron identificadas a partir de la base de datos proporcionada por la clínica Comfamiliar Risaralda. Para esto se utilizaron los códigos de la Clasificación Única de Procedimientos en Salud (CUPS) del Ministerio de Salud de Colombia, y se buscaron los CUPS específicos para HTL. Todas las pacientes fueron inicialmente evaluadas en la Unidad de Laparoscopia Ginecológica Avanzada y Dolor Pélvico - ALGIA, ubicada en la ciudad de Pereira, Colombia. Esta unidad se encarga de la realización de todos los procedimientos de endoscopia ginecológica por patología benigna en la clínica Comfamiliar, tanto para el tema asistencial como para el programa de entrenamiento. La técnica quirúrgica se realiza de manera estandarizada, según lo descrito por Einarssor y Suzuki (13).

Una vez identificadas las historias clínicas de las pacientes de la población del estudio, se recolectó la información en formulario de Excel específicamente diseñado para este estudio; se tomó información de las variables sociodemográficas, clínicas basales, los hallazgos intraoperatorios y las complicaciones intra y posoperatorias. Los datos de la variable de sangrado intraquirúrgico fueron extraídos de la descripción quirúrgica realizada por el cirujano, de la anotación del récord anestésico o de la nota de enfermería con respecto al volumen obtenido en el liner de la succión después de la cirugía. El peso uterino se tomó de la descripción quirúrgica o 
del resultado de la patología de la pieza quirúrgica. El seguimiento se hizo a partir de las notas de los controles médicos posoperatorios, las consultas ambulatorias, hospitalarias y de urgencias de los 30 días siguientes a la intervención. Para evaluar la calidad de la información se tomó aleatoriamente el 10\% de los datos y se compararon los datos digitados contra los documentos fuente. Además, se verificó la existencia los datos extremos y los faltantes en el archivo en Excel.

Variables medidas: edad, antecedentes ginecobstétricos, antecedentes quirúrgicos, diagnósticos prequirúrgicos, datos quirúrgicos (tiempo quirúrgico en minutos, sangrado intraquirúrgico en centímetros cúbicos y peso uterino en gramos), estancia hospitalaria en horas, complicaciones intra y posoperatorias según la clasificación Clavien-Dindo (14), complicaciones posquirúrgicas medidas hasta 30 días después de la HTL, días de incapacidad médica y mortalidad.

Análisis estadístico. Para el análisis de los datos se utilizó estadística descriptiva. Para las variables cuantitativas se calculó la mediana con la desviación estándar y para las variables cualitativas se realizaron tablas de frecuencias. Para el procesamiento de datos se utilizó Stata versión 14.0

Aspectos éticos. Antes de la cirugía todas las pacientes leyeron y firmaron el consentimiento informado, donde estaba incluido que algunos de sus datos podrían ser usados para trabajos de investigación. Este estudio fue aprobado por el Comité de Ética de la Clínica Comfamiliar. Se garantizó la confidencialidad y privacidad de la información. Acta 000032, aceptado el 7 de noviembre de 2017.

\section{RESULTADOS}

De acuerdo con los datos suministrados por la oficina de estadística de la Clínica Comfamiliar, en el periodo de estudio se realizaron 2.879 histerectomías por patología benigna, de estas se identificaron 1.513 HTL (52\%). Médicos externos al grupo de ALGIA realizaron 124 (8\%), por lo que no se incluyeron, y 39 (2,5\%) fueron excluidas por patología maligna. Finalmente, en el estudio se incluyeron 1.350 pacientes. El 98,5\% de estas se evaluaron entre los 7 y 14 días posoperatorio, y el 96,5\% nuevamente entre 30 y 45 días después.

Las pacientes con HTL tenían una edad media de 43,5 años (DE \pm 7 ), distribuidas por estratos, así: < 30 años (25\%), 31-40 años (30,2\%), 41-50 años (56\%), 51-60 años (9\%) > 60 años (3\%) (tabla 1). Las indicaciones prequirúrgicas más frecuentes fueron: sangrado uterino anormal, 987 (73,1\%); miomatosis uterina, 820 (60,7\%); adenomiosis, 791 (58,5 \%); dolor pélvico crónico, 639 (47,3\%); endometriosis, 284 (21,4\%); hiperplasia endometrial, 91 $(6,7 \%)$ y prolapso genital 14 (1\%). El $10 \%$ de las pacientes tenía un único diagnóstico, el $35 \%$ tenía dos diagnósticos y el 55 \% tenía 3 o más diagnósticos asociados a la histerectomía. El tiempo quirúrgico fue $95 \mathrm{~min}$ (DE \pm 31 ), el peso uterino tuvo una media de $236 \mathrm{~g}$ (DE \pm 133), el sangrado intraoperatorio

Tabla 1.

Datos sociodemográficos y clínicos de pacientes sometidas a histerectomía laparoscópica total en la clínica Confamiliar de Pereira por el Grupo ALGIA. Colombia, 2007-2017

\begin{tabular}{|l|c|c|c|}
\hline & Media & DE & Intervalo \\
\hline Edad & 43,5 & 7 & $18-86$ \\
\hline Embarazos & 2 & 1,3 & $0-12$ \\
\hline IMC & 27,6 & 2,4 & $24-39$ \\
\hline Número de cirugía abdominal previa & 0,54 & 0,8 & $0-9$ \\
\hline
\end{tabular}

DE: desviación estándar. 
$88 \mathrm{cc}(\mathrm{DE} \pm 66)$, la estancia hospitalaria 13 horas (DE \pm 20$)$ y la incapacidad médica 13 días (DE \pm 3 ).

Se presentaron complicaciones menores (grado I-II) en 49 (3,48\%) pacientes, y complicaciones mayores (grado III-V) en 36 pacientes (2,5\%); tres pacientes requirieron conversión a laparotomía (0,23\%). No hubo mortalidad en la muestra estudiada. Las complicaciones menores fueron: infecciones del tracto urinario bajo diagnosticadas en 14 pacientes por un urocultivo positivo y en otras 10 por sintomatología de infección urinaria baja. Todas recibieron tratamiento antibiótico ambulatorio según urocultivo y evolucionaron satisfactoriamente sin complicaciones. Un total de 11 pacientes requirió transfusión sanguínea $(0,81 \%)$; tres pacientes con trastorno de la coagulación requirieron manejo en conjunto con hematología y se transfundieron después de la cirugía; en dos de las pacientes la técnica quirúrgica fue difícil por endometriosis profunda y presentaron sangrado intraoperatorio que requirió transfusión de hemoderivados; cuatro pacientes tenían antecedente de sangrado uterino anormal con hemoglobinas previas bajas y fueron transfundidas en el intraoperatorio; dos pacientes se transfundieron luego de ser laparoconvertidas. Todas evolucionaron adecuadamente y fueron dadas de alta sin complicaciones. Nueve pacientes reconsultaron por dolor en los siguientes 7 días del posoperatorio (0,6\%); 5 de estas tenían antecedente de dolor pélvico crónico. Luego de descartar complicaciones posoperatorias mayores, las pacientes fueron manejadas con analgésicos en el servicio de urgencias y se les dio egreso para continuar manejo ambulatorio.

En cuanto a las complicaciones mayores, se presentaron 12 lesiones vesicales $(0,9 \%)$ y dos lesiones ureterales $(0,17 \%)$. En una paciente se sospechó lesión térmica ureteral izquierda con energía bipolar al acceder al pedículo inferior. En el mismo acto operatorio se decidió insertar catéter JJ que se dejó por 8 semanas con buena resolución del episodio y sin secuelas. La otra paciente presentó sección con tijera del tercio distal del uréter izquierdo durante el tratamiento de endometriosis profunda, se hizo diagnóstico intraoperatorio y se realizó anastomosis termino-terminal del uréter por laparoscopia e inserción de catéter JJ en el mismo acto quirúrgico. El retiro del catéter se realizó a las 12 semanas, sin complicaciones. Dos pacientes presentaron lesión intestinal $(0,17 \%)$; una presentó enterotomía de íleon distal al realizar liberación de adherencias densas a la pared pélvica por cirugía previa; se realizó rafia de la lesión por laparoscopia en dos planos y manejo intrahospitalario por dos días, con evolución adecuada y sin secuelas. La otra paciente presentó despulimiento de la serosa del recto sigmoides al liberarlo de la pared posterior del útero por endometriosis profunda; se realizó rafia de la serosa por laparoscopia y salida a las 24 horas sin complicaciones ni secuelas. Hubo un tromboembolismo pulmonar $(0,07 \%)$ que requirió manejo médico inicialmente en cuidado intermedio y luego en hospitalización de donde egresó 10 días después con anticoagulación ambulatoria y adecuada evolución clínica.

En 24 pacientes $(1,7 \%)$ se presentó infección del sitio operatorio. Cinco pacientes presentaron infección superficial (0,37\%); de estas, tres fueron manejadas ambulatoriamente con antibiótico (cefalosporinas de segunda generación) por vía oral, las dos restantes requirieron inicialmente manejo antibiótico con oxacilina parenteral intrahospitalario y luego manejo oral con dicloxacilina o cefalosporinas de segunda generación, ambulatorio. Todas completaron 7 días de tratamiento con buena evolución. Otras 19 pacientes presentaron absceso de cúpula vaginal $(1,41 \%)$ entre el tercer y décimo día posoperatorio, seis pacientes fueron llevadas a drenaje en el quirófano (dos por laparoscopia y cuatro por vía vaginal) y manejo antibiótico intrahospitalario por 72 horas; las 13 restantes fueron manejadas con antibiótico intrahospitalario por 48 a 72 horas. Todas las pacientes completaron manejo antibiótico ambulatorio por 7 días con evolución satisfactoria (tabla 2).

En cuanto a las tres pacientes laparoconvertidas, una de ellas tenía un útero poco móvil, de $1.650 \mathrm{~g}$, 


\begin{tabular}{|c|c|c|}
\hline \multirow[t]{2}{*}{ Complicaciones } & n (\%) & Clavien-Dindo \\
\hline & \multicolumn{2}{|c|}{ Menores } \\
\hline Infección urinaria & $24(1,7)$ & II \\
\hline Sangrado/Transfusión & $11(0,81)$ & II \\
\hline Reconsulta/Dolor/Fiebre & $9(0,6)$ & I \\
\hline Infección del sitio operatorio & $5(0,37)$ & II \\
\hline Total & $49(3,48)$ & \\
\hline \multicolumn{3}{|c|}{ Mayores } \\
\hline Absceso de cúpula & $19(1,4)$ & III a \\
\hline Lesión vesical & $12(0,8)$ & III b \\
\hline Lesión ureteral & $2(0,14)$ & III b \\
\hline Lesión intestinal & $2(0,14)$ & III b \\
\hline Tromboembolismo & $1(0,074)$ & IV a \\
\hline Total & $36(2,5)$ & \\
\hline Laparoconversión & $3(0,23)$ & \\
\hline
\end{tabular}

por lo que no fue posible acceder de manera segura a los pedículos inferiores y se decidió convertir a laparotomía. En otra paciente fue imposible el retiro de la pieza a través de la vagina por tamaño y deformidad del útero, que pesó $900 \mathrm{~g}$. La última paciente tenía gran mioma fúndico e intraligamentario de 18 cm y un útero de $680 \mathrm{~g}$ que requirió laparotomía para su extracción segura.

\section{DISCUSIÓN}

Este estudio describe el desempeño de un equipo quirúrgico que realizó 1.350 histerectomías durante 10 años en pacientes con patología ginecológica benigna. Un total de 85 pacientes $(5,9 \%)$ presentó complicaciones; 49 pacientes $(3,48 \%)$ presentaron complicaciones menores (Clavien-Dindo I-II). Las complicaciones mayores (Clavien-Dindo III-V) corresponden a 44 (3,2\%). No se presentó mortalidad en la población estudiada (14).
Respecto a la frecuencia general de complicaciones, nuestros resultados se encuentran dentro de los valores reportados por Lee et al. (5) quienes, en una revisión sistemática de ensayos controlados aleatorizados, que compara la HV con la HLT, informa que la frecuencia total de complicaciones en la HTL tuvo un rango entre 0 y $41 \%$ (7 estudios y 228 pacientes). Barber et al. reportaron una frecuencia de transfusión entre 2,5 y 4,3\% en pacientes sometidas HTL (10). La frecuencia de complicaciones es inferior a la informada en Colombia; De los Ríos et al., en un estudio retrospectivo en 837 pacientes, indica una tasa total de complicaciones del 12,5\%, de las cuales 3,1\% fueron mayores (15). Otro estudio hecho en Colombia informa que no hubo complicaciones mayores y una frecuencia de complicaciones tipo I del 9\% (16), que también se encuentra en el rango informado por Lee et al. (5). Un estudio de bases de datos que incluyó 441 
hospitales en Estados Unidos, realizadas entre 2007 y 2010, comparó la cohorte de mujeres expuesta a histerectomía asistida por laparoscopia con la HTL y reporta una frecuencia de complicaciones global del 5,5 y 5,3\% respectivamente (17).

Nuestro tiempo quirúrgico promedio fue de 95 min (DE \pm 31 ), comparable con lo publicado por Lee et al. (5) en su revisión sistemática, en la que los ECAS incluidos tuvieron tiempos que variaron entre $66(\mathrm{DE} \pm 14)$ y $114(\mathrm{DE} \pm 31) \mathrm{min}$. Por su parte, De los Ríos informa un tiempo quirúrgico de 85,9 min (DE \pm 30 ) (15) y Navarro anota una mediana de 60 min (rango: 45-120) (16).

La necesidad de transfusión en el 0,8\% de nuestra cohorte y en el 0,85\% en la cohorte de De los Ríos (15) es inferior a la reportada por Wright en los hospitales de Estados Unidos, con el 1,8 \% (17), y en la revisión sistemática de Lee que incluye el estudio de Allam, en la que el $3 \%$ requirió transfusión (5).

Como limitaciones del estudio se encuentran la metodología de cohorte retrospectiva; y que nuestros resultados son de una sola institución, por lo cual solo podemos inferir las conclusiones para esta población. Como fortalezas tenemos el importante número de pacientes intervenidas y su seguimiento.

\section{CONCLUSIÓN}

La histerectomía total por laparoscopia, realizada por el grupo ALGIA en la clínica Comfamiliar, es un procedimiento seguro, que está de acuerdo con los resultados publicados en la literatura internacional, ya sea en experimentos clínicos y cohortes de pacientes. Es importante que se sigan haciendo estudios prospectivos con criterios objetivos de evaluación para comparar el desempeño de los diferentes grupos e instituciones que ofrecen procedimientos quirúrgicos y entrenamiento.

\section{REFERENCIAS}

1. Gante I, Medeiros-Borges C, Águas F. Hysterectomies in Portugal (2000-2014): What has changed? Eur J Obstet Gynecol Reprod Biol. 2017;208:97-102. http://doi.org/10.1016/j.ejogrb.2016.11.021.
2. Fingar KR, Stocks C, Weiss AJ, Steiner CA. Most frequent operating room procedures performed in U.S. hospitals, 2003-2012: Statistical Brief \#186. In: Healthcare Cost and Utilization Project (HCUP) Statistical Briefs. Rockville (MD): Agency for Healthcare Research and Quality (US); 2014.

3. Turner LC, Shepherd JP, Wang L, Bunker CH, Lowder JL. Hysterectomy surgery trends: A more accurate depiction of the last decade? Am J Obstet Gynecol. 2013;208(4):277.e1-277.e2777. http://doi. org/10.1016/j.ajog.2013.01.022.

4. Reich H, DeCaprio J, McGlynn F. Laparoscopic hysterectomy. J Gynecol Surg. 1989;5:213-6. http://doi. org/10.1089/gyn.1989.5.213.

5. Lee SH, Oh SR, Cho YJ, Han M, Park JW, Kim SJ, et al. Comparison of vaginal hysterectomy and laparoscopic hysterectomy: A systematic review and meta-analysis. BMC Womens Health. 2019;19(1):83. http://doi. org/10.1186/s12905-019-0784-4.

6. Aarts JW, Nieboer TE, Johnson N, Tavender E, Garry R, Mol BW, Kluivers KB. Surgical approach to hysterectomy for benign gynaecological disease. Cochrane Database Syst Rev. 2015;(8):CD003677. http://doi. org/10.1002/14651858.CD003677.pub5.

7. Dedden SJ, Geomini PMAJ, Huirne JAF, Bongers MY. Vaginal and laparoscopic hysterectomy as an outpatient procedure: A systematic review. Eur J Obstet Gynecol Reprod Biol. 2017;216:212-23. http://doi. org/10.1016/j.ejogrb.2017.07.015.

8. Hwang JL, Seow KM, Tsai YL, Huang LW, Hsieh BC, Lee C. Comparative study of vaginal, laparoscopically assisted vaginal and abdominal hysterectomies for uterine myoma larger than $6 \mathrm{~cm}$ in diameter or uterus weighing at least $450 \mathrm{~g}$ : A prospective randomized study. Acta Obstet Gynecol Scand. 2002;81(12):1132-38. http://doi.org/10.1034/ j.1600-0412.2002.811206.x.

9. Mäkinen J, Brummer T, Jalkanen J, Heikkinen AM, Frasser J, Tomas E, et al. Ten years of progress-improved hysterectomy outcomes in Finland 19962006: A longitudinal observation study. BMJ Open. 2013;3(10):e003169. http://doi.org/10.1136/bmjopen-2013-003169. 
10. Barber EL, Harris B, Gehrig PA. Trainee participation and perioperative complications in benign hysterectomy: The effect of route of surgery. Am J Obstet Gynecol. 2016;215(2):215.e1-215.e2157. http://doi. org/10.1016/j.ajog.2016.02.022.

11. Driessen SR, van Zwet EW, Haazebroek P, Sandberg EM, Blikkendaal MD, Twijnstra AR, et al. A dynamic quality assessment tool for laparoscopic hysterectomy to measure surgical outcomes. Am J Obstet Gynecol. 2016;215(6):754 e1-754 e8. http://doi. org/10.1016/j.ajog.2016.07.004.

12. Alfa-Wali M, Osaghae S. Practice, training and safety of laparoscopic surgery in low and middle-income countries. World J Gastrointest Surg. 2017;9(1):1318. http://doi.org/10.4240/wjgs.v9.i1.13

13. Einarsson JI, Suzuki Y. Total laparoscopic hysterectomy: 10 steps toward a successful procedure. Rev Obstet Gynecol. 2009;2(1):57-64.

14. Dindo D, Demartines N, Clavien PA. Classification of surgical complications: A new proposal with evaluation in a cohort of 6336 patients and results of a survey. Ann Surg. 2004;240(2):205-13. http://doi. org/10.1097/01.sla.0000133083.54934.ae.

15. De los Ríos JF, Castañeda JD, Calle GA, Serna E, Vásquez RA, Arango AM, et al. Histerectomía laparoscópica total en la unidad de endoscopia ginecológica de la Clínica del Prado, Medellín (Colombia), 20022008. Rev Colomb Obstet Ginecol. 2009;60(4):3207. https://doi.org/10.18597/rcog.314

16. Navarro-Newball H. Histerectomía laparoscópica: Sistematización del procedimiento y propuesta para programas de capacitación. Colomb Med. 2005;36(2):115-9. Disponible en: http://www.scielo. org.co/scielo.php?script $=$ sci_arttext\&pid $=$ S165795342005000200009\&lng $=\mathrm{en}$
17. Wright JD, Ananth CV, Lewin SN, Burke WM, Lu YS, Neugut AI, et al. Robotically assisted vs laparoscopic hysterectomy among women with benign gynecologic disease. JAMA. 2013;309(7):689-98. http://doi. org/10.1001/jama.2013.186.

\section{CONTRIBUCIÓN DE LOS AUTORES}

Claudia Bastidas-Guarín: recolección de datos, análisis de datos, redacción del manuscrito, revisión y aprobación final del documento.

Claudia Patricia Zambrano-Moncayo: recolección de datos, valoración de pacientes, revisión y aprobación final del documento.

Jorge Darío López-Isanoa: valoración de pacientes, realización de la cirugía, redacción del manuscrito, revisión y aprobación final del documento.

José Duván López-Jaramillo: valoración de pacientes, realización de la cirugía, redacción del manuscrito, revisión y aprobación final del documento.

Ana Lucía Herrera-Betancourt: recolección de datos, realización de las cirugías, revisión y aprobación final del documento.

Angélica Cuello-Salcedo: redacción del manuscrito, revisión y aprobación final del documento.

Eliana Gutiérrez-Calderón: recolección de datos, revisión y aprobación final del documento.

Dany Leandro Piedrahíta-Gutiérrez: redacción del manuscrito, realización de la cirugía, revisión y aprobación final del documento.

Juan Diego Villegas-Echeverri: valoración de pacientes, realización de la cirugía, análisis de datos, redacción del manuscrito, revisión y aprobación final del documento. 